\title{
Die Aktionsart in der deutschen Sprache: Eine kategoriale Unterteilung ${ }^{*}$
}

The type of action in the German language: a categorial division

\author{
Rafael LÓPEZ-CAMPOS BODINEAU ${ }^{1}$ \\ Universität Sevilla (Spanien) / Universität Ateneum
}

\begin{abstract}
Das Ziel dieses Artikels ist es, einige der grundlegenden Konzepte im Zusammenhang mit der kontroversen Problematik der Aktionsart in der deutschen Sprache zu untersuchen. Konkret bedeutet dies den Vorschlag einer Gliederung der traditionellen Kategorien in zwei grundlegende Bereiche (Handlungen des Zustands/Handlungen des Zustandswechsels), die wiederum nach der ingressiven, durativen und egressiven Dimension der deutschen Verben unterteilt werden. All dies wird durch das Einbeziehen einer zusätzlichen, als „iterativ“ bezeichneten Kategorie vervollständigt, die mit Hilfe grammatikalischer Mittel, die nicht notwendigerweise verbaler Natur sind, zum Ausdruck gebracht wird.

Schlüsselwörter: Verb - Aktionsart - Temporalität - Kausalität - Verbzusätze
\end{abstract}

\begin{abstract}
The aim of this paper is to review some of the fundamental concepts concerning the widely discussed controversy on the mode of action in German. Specifically, the proposal articulates the traditional categories into two basic branches (actions of state / actions of change of state)
\end{abstract}

\footnotetext{
* Beim vorliegenden Artikel handelt es sich um die nach der entsprechenden inhaltlichen und bibliografischen Prüfung erstellte deutsche Übersetzung und Überarbeitung des 2010 in der Zeitschrift Futhark (Sevilla) veröffentlichten Arbeit mit dem Titel „El modo de acción en lengua alemana: una subdivisión categorial“.

1 https://orcid.org/0000-0001-8482-4469

Universidad de Sevilla, Departamento de Filología Alemana

Ateneum-Szkoła Wyższa w Gdańsku, Wydział Neofilologiczny

rafali@us.es
} 
subdividing the groups into the ingressive, durative and egressive dimensions of German verbs. The whole set is completed by an additional category called "iterative" which is shown by means of grammatical devices whose nature is not necessarily verbal.

Keywords: Verb - Mode of action - Temporality - Causality - Verb Prefixes

\section{Eingrenzung und Problemstellung}

Zusätzlich zu den grammatischen Kategorien Person, Numerus, Tempus, Aspekt, Modus und Genus Verbi ist es üblich, den (deutschen) Verben eine weitere Kategorie semantischer Art zuzuordnen, die sich mittels üblicherweise als „ingressiv“, „egressiv“, „durativ“, „punktuell“, „resultativ“, etc. bezeichneten Werten, auf die Aktionsart (AA) des Verbs beziehen. Im Gegensatz zu den vorhergehenden, ist diese Kategorie tendenziell a priori (LYONS, 1977: 639-640) abgrenzbar, sodass die Zuordnung eines dieser Kennzeichen zu einem konkreten Verb innerhalb seines ganzen Paradigmas konstant bliebt, ohne dass eine etwaige Beugung des Verbs die ursprünglich festgelegte Beschreibung verändert.

Wie aus den für diese Arbeit herangezogenen Quellen hervorgeht (EGG, 1994 und KRIFKA, 1989), ist die Berücksichtigung dieser Werte unseres Erachtens nach für die Beschreibung der semantischen Mikrostruktur der (deutschen) Verben unerlässlich, obwohl wir der Ansicht sind, dass nicht immer von einer Ausgangsklassifikation ausgegangen wird, welche die Gesamtheit der verfügbaren Einheiten innerhalb irgendeiner bestimmten lexikalisch-semantischen Koordinate abdeckt, noch eine monolithische Anordnung der bestehenden Terminologie festgelegt wird. In diesem Zusammenhang ist, um ein Beispiel zu geben, der uneindeutige Gebrauch (FEFILOV, 1981: 147) unter anderem der Zusammensetzungen ingressivichoativer, egressiv-terminativer, punktuell-kompletiver Art zu betrachten.

Ziel dieser Arbeit ist es daher, 1) eine Anordnung der wichtigsten Werte im Zusammenhang mit dem komplexen Gefüge der AA in der deutschen Sprache darzulegen, um unter diesem Gesichtspunkt in kohärenter Form das gesamte Verbalkorpus einer Sprache, statt bloß die Prototypen eines jeden der genannten Werte, zu analysieren und 2) mittels dieser Klassifikation die bestehende Nomenklatur anhand einer Eingrenzung der in diesem Bezug relevantesten semantischen Koordinaten zu systematisieren. 


\section{Kategorische Unterteilung}

Um die AA zu analysieren, ist das Umfeld von Temporalität und Kausativität auf direkte oder indirekte Art zu berücksichtigen. In beiden Fällen handelt es sich um semantische Felder, die mehr oder weniger stark den Handlungsablauf der Aktion als solche definieren. Auch sind beide ${ }^{2}$ bei der Beschreibung der Bedingungen zeitlicher Art, in denen die Handlung ausgeführt wird, sowie bei deren Verortung innerhalb einer zeitlichen Koordinate, mit oder ohne die Berücksichtigung von Relativitätsparametern, von großer Bedeutung. ${ }^{3}$

\subsection{Temporalität}

Die erste Dimension, die mit der Benennung der AA im Zusammenhang steht, umfasst den streng temporalen Geltungsbereich und ist in diesem Sinne notwendig, um zwischen Handlungen punktueller und durativer Natur und innerhalb dieser Dimensionen zwischen ingressiven, prozessualen und egressiven Handlungen zu unterscheiden (FEFILOV, 1981: 147-149). All diese können wiederum als iterativ oder nicht iterativ begriffen werden. Schlussendlich ergibt sich daraus folgendes:

\footnotetext{
2 TROST (1977: 2) bezieht außerdem den Aspekt des Verbs mit ein.

${ }^{3}$ Im Gegensatz zu dem, was KIEFER (1992: 92) bzw. EGG (1994) im Allgemeinen in Bezug auf die Anwendung dieses Problems auf die deutsche und ungarische Sprache formuliert haben, sind wir der Meinung, dass diese beiden Bereiche eine volle funktionale, strukturelle und semantische Autonomie genießen. Insofern es möglich ist, eine weitgehende Kompatibilität zwischen dem großen Teil der von jeder einzelnen dieser beiden Dimensionen abgeleiteten Varianten festzustellen - was in dieser Arbeit dargelegt werden wird.
} 

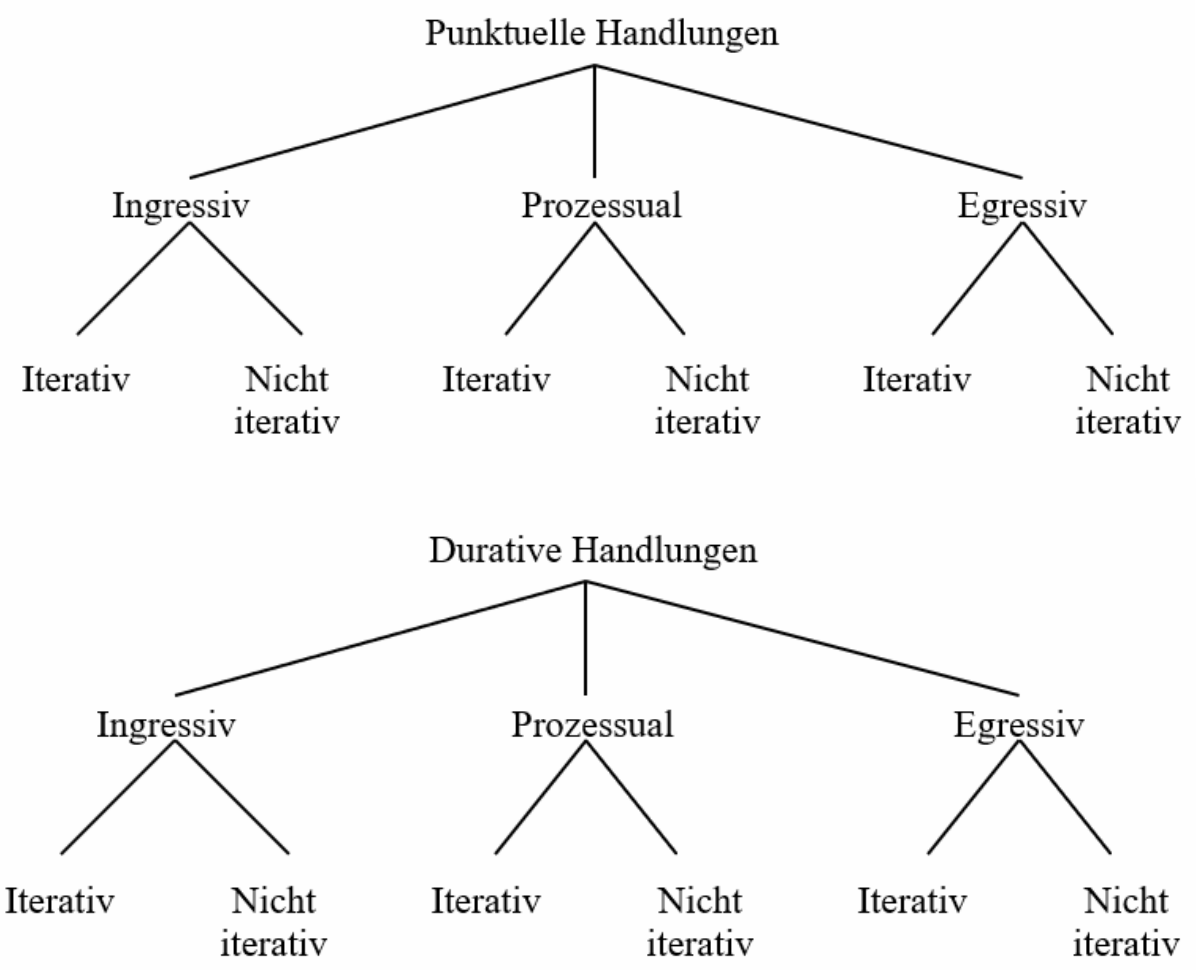

Der „punktuelle“ Wert (PUNKT) kann Verben zugeordnet werden, deren Handlung nicht mittels einer bestimmten Dauer quantifizierbar ist, insofern als dass die Handlung selbst ihren Anfang und ihr Ende darstellt. Beispiele dafür sind Verben wie finden, sterben, platzen, etc., die sich alle auf Handlungen beziehen, bei deren wörtlicher Darstellung es nicht möglich ist, durch Anfang und Abschluss irgendeine Art von Entwicklung zu erkennen (STEINITZ, 1981: 85). In diesen Fällen handelt es sich, wie im Zusammenhang mit der kausalen Koordinate erkennbar werden wird, um Verben des Zustandswechsels ${ }^{4}$, weil sie die Durchführung der betreffenden Handlung als einen Übergang implizieren Zust. A > Zust. B, zum Beispiel suchen > gefunden haben, etc.

In diesem Sinne kann von einem „punktuell prozessualen“ Charakter (PUNKT PROZESS) der Verben gesprochen werden, indem die beiden vom Zustandswechsel betroffenen Handlungen in ihrer Gesamtheit, oder zumindest ohne direkten oder indirekten Bezug zu der Anfangsphase der Handlung

${ }^{4}$ Dies bedeutet nicht, dass alle als ZUSTW. gekennzeichneten Verben punktuell sein müssen, wie wir bei der genaueren Untersuchung der kausalen und temporalen Koordinaten sehen werden. 
A und/oder der Endphase der Handlung B, betrachtet werden. Das Gegenteil ist der Fall, wenn sich das Verb auf den Beginn einer Handlung bezieht, die in einem späteren, mehr oder weniger langwierigen Prozess, fortgesetzt wird oder auf deren Endphase, wobei dann von PUNKT INGRESS oder PUNKT EGRESS Verben gesprochen werden kann. Siehe:

(1) nach dem schönen Tag am Strand schlief das Kind schnell ein

(2) Margaritte rauchte ihre Zigarette auf

Unter (1) handelt es sich um eine PUNKT-INGRESS-Handlung, da es sich A) um ein Verb des Zustandswechsels handelt, das sich auf eine Handlung bezieht, deren Erfüllung den Beginn und den Abschluss derselben bedeutet und B), da das abtrennbare Präverb ein einen Bezug zum Beginn der Handlung B herstellt, die durch die Basis des Verbs ausgedrückt wird. Genau das Gleiche tritt im Falle von (2) auf, wobei sich bei diesem Beispiel das Präverb auf das Ende der Handlung A bezieht, welche auch in diesem Fall durch die Basis des Verbs ausgedrückt wird. So bezieht sich die verbale Basis eines PUNKTINGRESS-Verbs immer auf den Zustand, der erreicht wird, während die als PUNKT-EGRESS gekennzeichneten sich auf den beziehen, von dem ausgegangen wird.

Daher ist die Analyse des abtrennbaren Präverbs, das bei jedwedem PUNKT-Verb vorhanden ist, sowie die Berücksichtigung seiner ursprünglichen semantischen Merkmale bei der Klärung der Frage, ob es sich um eine Handlung ingressiver oder egressiver Art handelt, von großer Bedeutung. Auf der anderen Seite handelt es sich bei als PUNKT gekennzeichneten Verben, die ohne jegliches Präverb auftreten, um prozessuale Bereiche generischer Art (siehe erneut die Verwendung von finden, sterben, etc.).

Handlungen ingressiver oder egressiver Art (wie die bei einschlafen oder aufrauchen behandelten) müssen grundsätzlich dem PUNKT-Wirkungsbereich der Verben zugeordnet werden, da sie sich auf den Punkt des (zeitlich nicht ausdehnbaren) Wechsels beziehen, durch den von einem Zustand in einen anderen übergegangen wird. Dennoch müssen wir diese Varianten gleichermaßen in Bezug auf die durativen Verben (DURAT) betrachten, da es gelegentlich möglich ist, diesen eine gewisse Weiterführung zuzuordnen, die sich aus der Verwendung einer ursprünglichen Bedeutung im übertragenen Sinne ergibt (KRIFKA, 1989: 65). Ein Beispiel dafür ist die Möglichkeit jedes 
der vorher erwähnten Verben mit Adverbien wie langsam, allmählich, etc. zu verbinden, welche alle eine gewisse zeitliche Ausdehnung mit sich bringen: ${ }^{5}$

(3) nach dem schönen Tag auf dem Berg schlief das Mädchen langsam ein

(4) Andrea raucht ihre Zigarette langsam auf

In diesen Fällen handelt es sich um die gleichen Verben, die vorher noch als punktuell definiert wurden, obwohl sie unter diesen Umständen durch die Verwendung der adverbialen Komponente langsam eine durative Dimension erlangen. Es versteht sich von selbst, dass sie als solche Verben Einheiten des Typs PUNKT sind. Dies ergibt sich weiterhin und auch in diesen Fällen durch den punktuellen Charakter, den der durch diese Verben ausgedrückte Zustandswechsel darstellt, sowie durch die Unmöglichkeit diese mit adverbialen Komponenten eigentlich temporal-durativer Natur zu kombinieren (siehe auch die Verwendung der Komponente $x$ Stunde( $n$ ) lang, bei der Bildung von * er starb eine Stunde lang, ${ }^{*}$ er fand den Weg eine Stunde lang, ${ }^{*}$ er schlief zwei Stunden lang ein), obwohl diese in Beispielen wie (3) und (4) unmöglich ein anderer Zustand als DURAT zugewiesen werden kann. Dies erfolgt allerdings nicht in Anlehnung an deren Zustandswechsel an sich, sondern an die gelegentlich langsamere Annäherung an oder Abkehr von eben jenem Zustandswechsel.

Demzufolge wird den durativen Verben die gleiche Unterteilung zugewiesen werden, die auch in der Analyse der punktuellen Verben erfolgt ist, was sich in der Berücksichtigung der drei grundlegenden Varianten „ingressiver“ (DURAT INGRESS), „prozessualer“ (DURAT PROZESS) und „egressiver“ (DURAT EGRESS) Art widerspiegelt. Die Einordnung erfolgt dabei jeweils entsprechend der deskriptiven Bedeutung des Verbs, welches Gegenstand der Analyse ist und sich auf den Anfang, die Gesamtdauer oder das Ende der entsprechenden Handlung bezieht.

Die verbale Analyse aus der Perspektive dieser ersten Koordinate ist durch die Gegensätzlichkeit der Varianten INGRESS und EGRESS charakterisiert $^{6}$, sowie durch die Vereinbarkeit beider mit PROZESS. In diesem

\footnotetext{
${ }^{5}$ In keinem Fall dürfen wir die Kennzeichnung DURAT auf jene Verben reduzieren, welche die Darstellung einer vollständigen Handlung bedeuten. Auch die Anfangs- und Endperioden können in einer in der Zeit ausgedehnten Form durchgeführt werden.

${ }^{6}$ In diesem Sinne sei die Unmöglichkeit einer Vereinbarkeit der Varianten PUNKT und DURAT sowie der Varianten INGRESS und EGRESS beachtet. Wie dem auch sei, schließt dies die Abgrenzung vollständiger Paradigmen in dieser Hinsicht nicht aus, obwohl diese immer auf der Berücksichtigung verschiedener lexematischer Einheiten und/oder einer Einbeziehung des
} 
Sinne gibt es beispielsweise die Möglichkeit ein Verb des PROZESS-Typs als INGRESS-Typ zu begreifen, so möglich bei sich verlieben aufgrund seiner Verwandtschaft mit den Referenzverb (RV) lieben ${ }^{7}$, oder gleichermaßen ein INGRESS-Verb als PROZESS-Verb, wie etwa bei anfangen, sich zu verlieben: ${ }^{8}$

[sich verlieben]

[anfangen, sich zu verlieben]
PROZESS

INGRESS RV [lieben]

\section{PROZESS}

INGRESS
$\mathrm{RV}$ [sich verlieben]

Wie bereits erwähnt, ist in der deutschen Sprache die Bezugnahme zu INGRESS- und EGRESS-Handlungen sowie zu denen die als PROZESS gekennzeichnet werden insbesondere durch die Anwendung einiger abtrennbarer Präverben (STIEBELS, 1994: 72) üblich, denen wir eine präpositionale Grundlage zuschreiben. Konkreter gehören dazu $a b$, an, auf, aus, durch und ein, wobei diese sich nach der folgenden Komponentenanalyse aufteilen:

AcI-Verbs lassen (platzen[PUNT], platzen lassen[DURAT INGRESS], aufhören, etwas platzen zu lassen[DURAT EGRESS], etc.) beruhen muss.

${ }^{7}$ In diesem Sinne ergibt es sich die Betrachtung eines RV im Falle der als DURAT-PROZESS gekennzeichneten Verben als gleichermaßen interessant, da diese gemäß ihren jeweiligen Entsprechungen (Verben und/oder alternative Umschreibungen) INGRESS und EGRESS auch als gegensätzlich begriffen werden. Man betrachte dazu die PROZESS-Natur von schlafen gegenüber der INGRES-Natur von einschlafen, etc. Unabhängig davon und weil die Bezeichnung PROZESS im Allgemeinen innerhalb eines durativen Wirkungsbereichs die vorherrschende ist, wird eine Analyse dieser Eigenschaften niemals so notwendig sein wie im umgekehrten Fall.

${ }^{8}$ In Bezug auf alles bisher dargelegte möchten wir darauf hinweisen, dass die Modi INGRESS, PROZESS und EGRESS irgendeiner Handlung nicht immer eine Identifikation mit einer konkreten lexischen Einheit aufweisen müssen, weswegen gelegentlich auf Redewendungen wie „mit etwas anfangen“ / „mit etwas aufhören“ zurückgegriffen werden muss. Dazu eignet sich der Vergleich vom Paradigma eine Zigarette anrauchen (INGRESS) - eine Zigarette rauchen (PROCESS) - eine Zigarette aufrauchen (EGRESS), in dem alle drei Varianten zur Verfügung stehen, mit einem anderen wie den Ball anspielen (INGRESS) - den Ball spielen (PROZESS) damit aufhören, mit dem Ball zu spielen, in welchem wir wegen der Ermangelung einer EGRESS-Variante auf die Verwendung von aufhören zurückgreifen müssen. Der gleiche Mechanismus ist als egressive Variante vieler anderer ingressiver Verben produktiv (oder von Fall zu Fall bei durativen), wie zum Beispiel beim bereits erwähnten verliebt sein (er war ins Mädchen verliebt > er hörte auf, ins Mädchen verliebt zu sein). 


\begin{tabular}{|c|c|c|c|}
\hline Präverb & $\begin{array}{c}\text { PUNKT / DURAT } \\
\text { INGRESS }\end{array}$ & $\begin{array}{c}\text { PUNKT / DURAT } \\
\text { PROZESS }\end{array}$ & $\begin{array}{c}\text { PUNKT / DURAT } \\
\text { EGRESS }\end{array}$ \\
\hline$a b$ & - & - & + \\
\hline$a n$ & + & + & - \\
\hline$a u f$ & + & - & + \\
\hline aus & + & + & - \\
\hline durch & - & + & - \\
\hline ein & + & - & + \\
\hline
\end{tabular}

Siehe:

$\mathrm{ab}$ [+ egress]: abheilen, abglühen, abglimmen, abklingen, etc.

an [+ ingress]: anbraten, anrauchen, anzahlen, anheben, anwärmen, etc.

an [+ durat]: anhalten, andauern, etc.

auf [+ ingress]: aufblühen, aufatmen, aufschreien, aufglimmen, aufglühen,

etc.

auf [+ egress]: aufessen, auffressen, aufrauchen, aufbrauchen, aufzehren, etc.

aus [+ ingress]: ausbrechen, auslösen, etc.

aus [+ durat]: aushalten, etc.

aus [+ egress]: ausarbeiten, austrinken, auszementieren, auslernen, ausreden,

etc.

durch [+ durat]: durchlesen, durchdiskutieren, durchbacken, etc.

ein [+ ingres]: einschlafen, einfühlen, einleben, einarbeiten, etc.

Diese überaus weitreichende Verfügbarkeit, die in der deutschen Sprache existiert, stößt auf zwei grundlegende Einschränkungen:

A) Nicht immer kann die INGRESS-, PROZESS- oder EGRESS-Dimension der durch die verbale Basis bezeichneten Handlung mittels der Verwendung einiger dieser Präverben ausgedrückt werden. Dies ist beispielsweise beim EGRESS zu anspielen [INGRESS] / spielen [PROZESS] oder beim INGRESS zu reden [PROZES] etc. der Fall. Siehe dazu:

anspielenINGRESS $>$ spielenDURAT $>$ *ausspielenEGRESS / aufspielenEGRESS

${ }^{*}$ anredenINGRESS $>$ redenDURAT $>$ ausredenEGRESS

andeutenINGRESS $>$ deutenDURAT $>{ }^{*}$ ausdeutenEGRESS $/{ }^{*}$ aufdeutenEGRESS

B) Die Identifizierung eines dieser Präverben mit den dargestellten Werten erlaubt in keinem Fall eine rein zufällige Anwendung desselben. Sichtbar 
wird dies bei der Betrachtung der Unmöglichkeit, Vereinbarkeiten zwischen den INGRESS, PROZESS oder EGRESS aufzustellen:

$\begin{array}{ll}\text { anbratenINGRESS } & { }^{*} \text { einbratenINGRESS } \\ \text { durchbratenDURAT } & >{ }^{*} \text { anbratenDURAT } \\ \text { ausreden } \text { EGRESS }> & { }^{*} \text { aufreden } \text { EGRESS }\end{array}$

In Bezug auf diese zweite Einschränkung kann das Auftreten gewisser Konkurrenzen nicht ausgeschlossen werden, welche selten absolut anwendbar sind, da die eine oder andere Option normalerweise mit einem bestimmten semantischen oder sogar pragmatischen Wert verbunden ist. Besonders auffallend ist der Fall der Basis halten, die mit den drei als PROZESS gekennzeichneten Präverben in Verbindung gesetzt werden kann, wenn auch nie gleich geartet, bedingt durch die Heterogenität der drei lexikalischen Zusätze, deren Träger sie sind:

(5) eine Belastung, einen Stress durchhalten

(6) seine Nerven halten nicht lange aus

(7) der Regen hält an

Es versteht sich von selbst, dass sich viele der von den verschiedenen Präverben beigetragenen Bedeutungen in enger Beziehung zu denjenigen befinden, die den jeweiligen Homonymen adverbial-präpositionaler Natur innewohnen ${ }^{9}$, da es diese waren, aus denen sie sich im Laufe der Geschichte entwickelt haben. Daher ist es aus diesem Grunde auf die folgende Art und Weise möglich, Verbindungen zwischen den räumlichen und zeitlichen Dimensionen herzustellen:

\begin{tabular}{|c|c|}
\hline Räumliche Dimension & Zeitliche Dimension \\
\hline Innere Verschiebung & INGRESSIVER Wert \\
\hline Äußere Verschiebung & EGRESSIVER Wert \\
\hline Transversale Verschiebung & DURATIVER Wert \\
\hline
\end{tabular}

Die Möglichkeit jedwedes vorbestimmte Verb mittels eines der Präverben, die gemäß einer der beiden Dimensionen festgelegt wurden, neu $\mathrm{zu}$ interpretieren, erlaubt es die festgestellten Verbindungen $\mathrm{zu}$ belegen

\footnotetext{
9 Es wird sich stets auf trennbare Präverben bezogen, die als „Indikatoren der Aktionsart“ und nicht als „Modifikatoren adverbialen Typs“ begriffen werden, d. h. all jene Fälle in denen das Präverb eine mit den behandelten Werten assoziierte Kennzeichnung darstellt, getrennt von jenen ursprünglich präpositionaler und adverbialer Art.
} 
und so eine Analyse klarer kognitiver Nuancen vorzunehmen (STORSCH, 1978: 96). Dies ist beispielsweise in Ausdrücken wie ein Kind einsingen, ein neues Auto einfahren, sich einarbeiten, etc. zu erkennen:

(8 a) ein Kind einsingen

(8 b) ein Kind in den Schlaf bringen (räumliche Neuinterpr.)

(8 c) ein Kind durch Singen ins Schlafen bringen (zeitliche Neuinterpr.)

(9 a) ein neues Auto einfahren

(9 b) ein Auto ins Fahren bringen (räumliche Neuinterpr.)

(9 c) anfangen, ein Auto zu probieren (zeitliche Neuinterpr.)

Beziehungen ähnlicher Art können durch aus als Präverb in Bezug auf die Zuweisung einer „äußeren Verschiebung“ und aus als Kennzeichen der EGRESS-Aktionsart, durch durch als denotatives Präverb einer „transversalen Verschiebung" und durch als Kennzeichen einer PROZESSAktionsart, etc. ausgedrückt werden.

Im Rahmen der mittels trennbarer Präverben ausgedrückten temporalen Koordinate ist ebenso die bestehende Konkurrenz zwischen den behandelten Mechanismen und dem Gebrauch von anfangen und aufhören als prototypische Verben der INGRESS- und EGRESS-Handlungen hervorzuheben. Im Prinzip können wir diese Konkurrenz als absolut bewerten, obwohl es nicht wenige Fälle gibt, in denen es nicht möglich ist, den dafür notwendigen Substitutionstest durchzuführen (vgl. 10 und 11), oder solche, in denen ein solcher Test zwar möglich ist, jedoch erhebliche Bedeutungsverschiebungen mit sich bringt (vgl. 12 und 13):

(10) den Schrank anheben

(11) angefangen, den Schrank zu heben

(12) Kartoffeln anbraten

(13) anfangen, Kartoffeln zu braten

Diese Art des semantischen Unterschieds, insbesondere in Bezug auf Phänomene wie das durch das Paar (12) und (13) dargelegte, führt dazu, den Präverben, da sie als „Indikatoren der Aktionsart" ${ }^{10}$ konzipiert sind, im Allgemeinen nicht nur Werte zuzuordnen, die sich auf die Aktionsart selbst beziehen, sondern auch Nuancen unterschiedlichster Art, sodass es zur Substituierung des Präverbs gelegentlich notwendig ist, auf eine nicht

\footnotetext{
${ }^{10} \mathrm{Im}$ Gegensatz zu anderen, die als „Modifikator adverbialer Art“, „Präposition mit pronominaler oder postpositionaler Funktion“ bezeichnet werden.
} 
unerhebliche Menge von Komponenten adverbialer Art zurückzugreifen. Obwohl es sich explizit um Ausdrucksmechanismen der egressiven Variante handelt, ersetzen diese nicht immer die Präverben, denen eine solche Bedeutung innewohnt. Dies liegt daran, dass das Präverb, zusätzlich zu dessen Kennzeichen als EGRESS, Werte adverbialen Typs unterschiedlichster Natur, etc. ausdrückt. Erkennbar ist dies in Beispielen wie:

(14) er hat die Straße auszementiert

(15) er hat die Straße zu Ende zementiert

(16) er hat aufgehört, die Straße zu zementieren

Die Bedeutung von aus in (14) entspricht nicht exakt der von zu Ende in (15), beziehungsweise der aufhören innewohnenden in (16), da aus neben der lediglich temporalen Beziehung noch andere, ebenso wichtige, festlegt. Aus kognitiver Perspektive werden diese durch die Bedeutung von aus selbst, als Bestandteil eines ursprünglichen Charakters adverbial-präpositionaler Art bestimmt. Dies sorgt dafür, dass die Substituierung des Präverbs die Anwendung anderer, zusätzlicher adverbialer Elemente wie ganz und gar, vom Anfang bis zum Ende, etc. verlangt.

Besondere Aufmerksamkeit verdienen die Aktionsunterarten iterativ / nicht iterativ (ITERAT / NICHT-ITERAT), die im Allgemeinen sowohl auf PUNKT- als auch auf PROZESS-Handlungen anwendbar sind, bedingt durch die große Verfügbarkeit grammatikalischer Mechanismen in der deutsche Sprache gegenüber anderen Sprachen. In diesem Sinne ist die Produktivität von zurück (sich zurückversetzen, sich zurückmelden, etc.), nach (nachbügeln, nachlesen, etc.) oder über (übermalen, überstreichen, etc.) als Ausdrucksmittel der einfachen Iteration, sowie anderer Alternativen wie zum Beispiel die Anwendung von Suffixen wie -ln / - rn, wie sie von klingen > klingeln, lachen > lächeln, etc. (BALZER, 1999: 79) abgeleitet sind, zu beachten.

Wie wir bereits im Hinblick auf die INGRESS-, PROZESS- und EGRESSHandlungen festgestellt haben, ist es auch in Bezug auf die Zuweisung von Iterationen mittels Präverben nicht möglich eine absolute Produktivität derselben zu erreichen. Diesbezüglich sind die Eingrenzungen wie lieben > * nachlieben, hassen $>{ }^{*}$ nachhassen, etc. zu sehen. Noch problematischer ist der Ausdruck der Iteration mittels Präverben im Falle von Handlungen, die durch bereits zusammengesetzte Basen formuliert werden (anfangen, * nachanfangen.) oder bei der Bildung von Verbindungen mit in sich bereits iterativer Bedeutung (nachbügeln $>^{*}$ nachnachbügeln). In all diesen Fällen ist es üblich auf die Anwendung anderer Alternativmechanismen 
zurückzugreifen, beispielsweise pronominaler oder adverbialer Komponenten im Allgemeinen (kochen > zweimal kochen, nachbügeln > ein paar Mal nachbügeln, etc.), da die doppelte Präverbalisierung in der deutschen Sprache mit Einschränkungen verbunden ist.

In diesem Sinn ist hervorzuheben, dass die ITERAT-Handlungen, die mittels irgendeines der Mechanismen, die gerade aufgezeigt wurden, ausgedrückt werden, mit so vielen Iterationen verbunden werden können, wie unser kommunikatives Bedürfnis verlangt. Es reicht in solchen Fällen die Veränderung der verfügbaren Iterationsmechanismen, um die Doppelungen, wie bereits erwähnt, zu vermeiden. Beispielhaft dafür steht die Abfolge, ich habe das Hemd nachgebügelt $>$ ich habe das Hemd zweimal nachgebügelt > ich habe das Hemd noch zweimal nachgebügelt, welche auf folgende Weise dargestellt werden kann:

[nachbügeln] ITERAT

$$
\begin{aligned}
& \left.\left[[\text { zweimal }]_{\text {ITERAT }} \text { nachbügeln }\right]_{\text {ITERAT }}\right] \\
& {\left[\left[[\text { noch }]_{\text {ITERAT zweimal }}\right]_{\text {ITERAT }} \text { nachbügeln }\right]_{\text {ITERAT }}}
\end{aligned}
$$

Unabhängig vom letztendlich verwendeten Mechanismus präsentiert sich uns die Iteration als eine auf die verschiedenen festgelegten Untergruppen weitgehend anwendbare Variante. Siehe:

Verb PUNKT INGRESS NICHT-ITERAT: einschlafen

Verb PUNKT INGRESS ITERAT: wieder (etc.) einschlafen

Verb PUNKT PROZESS NICHT-ITERAT: etwas finden

Verb PUNKT PROZESS ITERAT: etwas wieder (etc.) finden

Verb PUNKT EGRESS NICHT-ITERAT: eine Zigarette aufrauchen

Verb PUNKT EGRESS ITERAT: eine Zigarette wieder (etc.) aufrauchen

Verb DURAT INGRESS NICHT-ITERAT: langsam einschlafen

Verb DURAT INGRESS ITERAT: wieder (etc.) langsam einschlafen

Verb DURAT PROZESS NICHT-ITERAT: nach Madrid fahren

Verb DURAT PROZESS ITERAT: wieder (etc.) nach Madrid fahren

Verb DURAT EGRESS NICHT-ITERAT: eine Zigarette langsam

aufrauchen

Verb DURAT EGRES ITERAT: eine Zigarette langs. wieder (etc.)

aufrauchen 
Entsprechend allem bisher ausgeführten muss jede Handlung innerhalb eines „zeitlichen Rahmens“ angesiedelt sein, der nicht nur die mit der zu analysierenden Handlung verbundene Variante, sondern auch die übrigen Aussagen umfasst. Viele von ihnen werden mit Hilfe der Anwendung der erwähnten unterschiedlichen grammatikalischen und lexikalischen Mechanismen erreicht, da es (in der deutschen Sprache) nicht ausreichend lexikalische Einheiten gibt, um die Gesamtheit der aufgeführten Möglichkeiten zu vervollständigen. Siehe in diesem Zusammenhang das Themenfeld, das sich auf einige der bereits erwähnten Aktionen bezieht, z. B. rauchen:

\begin{tabular}{|c|c|c|c|}
\hline \multicolumn{4}{|c|}{ KAUSALES THEMENFELD RAUCHEN } \\
\hline & $\begin{array}{c}\text { DURAT / PUNKT } \\
\text { INGRESS } \\
\end{array}$ & $\begin{array}{c}\text { DURAT / PUNKT } \\
\text { PROZESS }\end{array}$ & $\begin{array}{c}\text { DURAT / PUNKT } \\
\text { EGRESS } \\
\end{array}$ \\
\hline ITERAT & $\begin{array}{c}\text { anfangen zu rauchen } \\
\text { eine Zigarette } \\
\text { anrauchen, etc. }\end{array}$ & $\begin{array}{c}\text { eine Zigarette } \\
\text { rauchen }\end{array}$ & $\begin{array}{l}\text { aufhören, eine } \\
\text { Zigarette zu rauchen } \\
\text { (eine Zigarette) zu } \\
\text { Ende rauchen, } \\
\text { eine Zigarette } \\
\text { aufrauchen, etc. }\end{array}$ \\
\hline NICHT-ITERAT & $\begin{array}{l}\text { wieder anfangen, } \\
\text { eine Zigarette zu } \\
\text { rauchen } \\
\text { (eine Zigarette) } \\
\text { wieder anrauchen }\end{array}$ & $\begin{array}{l}\text { (eine Zigarette) } \\
\text { wieder rauchen }\end{array}$ & $\begin{array}{c}\text { wieder aufhören, } \\
\text { eine Zigarette zu } \\
\text { rauchen } \\
\text { eine Zigarette } \\
\text { wieder zu Ende } \\
\text { rauchen } \\
\text { eine Zigarette } \\
\text { (wieder) aufrauchen }\end{array}$ \\
\hline
\end{tabular}

\subsection{Kausalität}

Diese zweite substanzielle Dimension bezieht sich auf die „verursachte“ oder „nicht verursachte“ Bedeutung (KAUS / NICHT-KAUS), die durch die von dem $\mathrm{zu}$ analysierenden Verb beschriebene Handlung ausgedrückt wird. Innerhalb jeder dieser Varianten wiederum wohnt diesen eine Kontinuität / NichtKontinuität inne, die durch die Zuweisung der Kennzeichen „Zustand“ und „Zustandswechsel“ (ZUST / ZUSTWECHS) dargestellt wird. 
Im Hinblick auf die Unterscheidung KAUS / NICHT-KAUS ist weniger die Transitivität als vielmehr die Beteiligung eines tätigen Subjekts zu betrachten, da jedes Verb entweder als KAUS oder NICHT-KAUS bezeichnet wird. Dies liegt nicht unbedingt an den entsprechenden transitiven und intransitiven Werten, sondern an der eigentlichen Kausativitätsbeziehung.

Transitivität und Kausativität müssen daher als voneinander unabhängige kognitive Dimensionen aufgefasst werden (TROST, 1977: 8-10), was zur Zusammensetzung vierer möglicher Kombinationen führt:

(A) Verben TRANS, KAUS

(B) Verben TRANS, NICHT-KAUS

(C) Verben INTRANS, KAUS

(D) Verben INTRANS, NICHT-KAUS stellen, legen, setzen, etc.

bekommen, erhalten, kriegen, etc. antworten, berichten, helfen, etc. stehen, liegen, sitzen, etc.

Diese Autonomie der Transitivitäts- und Kausativitätsdimensionen ergibt sich besonders deutlich aus den als (B) und (C) gekennzeichneten Kombinationen ${ }^{11}$. In Bezug auf (B) handelt es sich um elative Verben, allesamt transitiv, aber nicht als KAUS gekennzeichnet, insofern als dass der Akteur der Handlung nicht so sehr mit dem grammatikalischen Subjekt als vielmehr mit einer impliziten Komponente des unpersönlichen Typs assoziiert wird. Viele andere Verben, die ebenfalls der Kombination (B) angehören, sind die PUNKT-Verben finden, begegnen, etc., deren semantische Mikrostruktur zusätzlich mangelnde Intentionalität als solche aufweist.

Ebenso auffällig in diesem Sinne ist die Kombination (C), in der die intransitive Natur eindeutig kausativer Verben wie antworten, berichten oder helfen erkannt wird. Sie alle zeigen den eindeutigen Gebrauch eines tätigen Subjekts, ohne jedoch als transitiv aufgefasst zu werden (jdm. auf etwas antworten $>{ }^{*} j d n$. antworten; $j d m$. von etwas berichten $>{ }^{*} j d n$. berichten; $j d m$ helfen $>{ }^{*}$ jdn helfen, etc.). ${ }^{12}$

Transitivität und Kausativität verhalten sich daher nicht immer proportional zueinander. Während sich die erste auf die Kategorie der Rektion und damit auf die syntaktische Dimension des Verbs im Satz bezieht, wirkt sich die zweite im Gegenteil eher auf das semantische Feld selbst aus. Demnach

\footnotetext{
11 (A) und (D) stellen eine Beziehung zwischen TRANS und KAUS / INTRANS und NICHTKAUS her, die beide in jeder Sprache prototypisch sind.

12 Die Kennzeichnungen TRANS / INTRANS stehen in keinem direkten Zusammenhang mit der Problematik der AA. Deren Behandlung in diesem Abschnitt dient nur dazu, eine Abgrenzung des Referenzfeldes der Werte KAUS / NICHT-KAUS vorzunehmen.
} 
kann die Abgrenzung der KAUS-Variante durch Umformulierung des zu analysierenden Satzes unter Verwendung von Verben wie verursachen oder bewirken erfolgen (siehe in diesem Sinne Umformulierungen wie sich hinsetzen > die Sich-Hinsetzen-Aktion absichtlich und bewusst verursachen / ausführen, sich hinstellen > die Sich-Hinstellen-Aktion absichtlich und bewusst verursachen / ausführen, sich hinlegen > die Sich-HinlegenAktion absichtlich und bewusst verursachen / ausführen), was auch bei intransitiven Verben möglich ist. Siehe:

(18) jdm. helfen > verursachen, dass jemand Hilfe bekommt

(19) jdm. antworten > verursachen, dass jemand eine Antwort bekommt

In jedem Fall wird immer ein zusätzliches Prädikat einbezogen, das sich auf die Wirkung der durch das Verb beschriebenen Handlung bezieht und welches als RESULTAT gekennzeichnet werden wird. Siehe:

(20 a) sich hinsetzen

(20 b) (absichtlich / bewusst) verursachen $[\text { sitzen }]_{\text {RESULTAT }}$

(21 a) einen Brief schreiben

(21 b) (absichtlich / bewusst) verursachen [der Brief ist geschrieben] RESULTAT

(22 a) einen Satz analysieren

(22 b) (absichtlich / bewusst) verursachen [der Satz ist analysiert $]_{\text {RESULTAT }}$

(23 a) jemanden besuchen

(23 b) (absichtlich / bewusst) verursachen, [jemand wird besucht $]^{13}$ RESULTAT

Andererseits leitet sich die Unterscheidung zwischen ZUST und ZUSTWECHS von der semantischen Grundlage des Verbs selbst ab, wobei die erste Variante in den Fällen angewendet werden kann, in denen die Ausführung der durch ein bestimmtes Verb beschriebenen Handlung, den Übergang zu einem

13 Das Konzept des Resultats muss weniger mit der Unterkategorie KAUS / NICHT-KAUS verbunden werden als mit der grundlegenden Kategorie ZUST / ZUSTWECHS. In diesem Zusammenhang steht die Verknüpfung von Verben wie zerbrechen mit diesen beiden Kategorien, mittels Paaren wie die Vase ist zerbrochen - man hat die Vase zerbrochen, was gleichermaßen auf den ganzen Bereich ausgeweitet werden kann (sägen, scheren, schneiden, etc.). Gleichermaßen resultativ und dementsprechend auch zur Gruppe ZUSTWECHS gehörend sind Verben aus dem Konstruktionsbereich (bauen, montieren, etc.) und vergleichbare. 
anderen Zustand bewirkt $\left(\mathrm{Z}_{1}>\mathrm{Z}_{2}\right)$ und die zweite Variante im umgekehrten Fall. Wird beispielsweise wieder von den Feldern „Platzierung“ (Platz [stellen, legen, setzen, etc.]) und „statische Positionierung“ (Posit [stehen, liegen, sitzen, etc.]) ausgegangen, stellt man eine klare Konzeption der ersteren als ZUSTWECHS und der letzteren als ZUST fest, dank der räumlichen Veränderung bzw. der Ruhe, die sie ausdrücken. Siehe:

$$
\begin{aligned}
& \text { LIEGEN }=\mathrm{Z}_{1}(\text { liegen })>\mathrm{Z}_{2}(\text { liegen }) \\
& \left.\mathrm{LEGEN}=\mathrm{Z}_{1}[\text { nicht liegen }(\text { stehen, etc. })]>\mathrm{Z}_{2} \text { (liegen }\right) \\
& \text { STEHEN }=\mathrm{Z}_{1}(\text { stehen })>\mathrm{Z}_{2}(\text { stehen }) \\
& \text { STELLEN } \left.=\mathrm{Z}_{1} \text { (nicht stehen }(\text { sitzen, etc. })>\mathrm{Z}_{2} \text { (stehen }\right)
\end{aligned}
$$

Diese Abgrenzung der Kennzeichen ZUST / ZUSTWECHS kann auf unzähligen Wortfeldern mittels einer Analyse ähnlicher Eigenschaften vorgenommen werden. Einige dieser Paare sind:

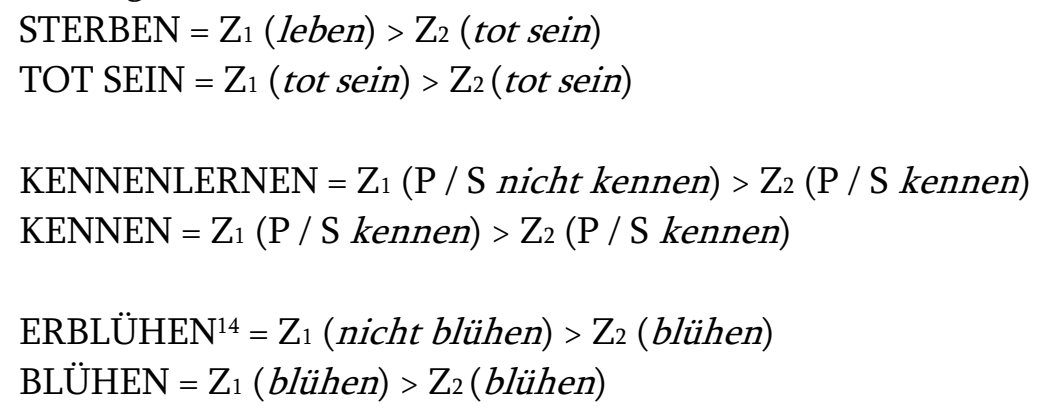

Diese Analyse zwingt uns natürlich dazu, bestimmten Verben beide Kennzeichnungen zuzuordnen, je nachdem ob sie die eine oder andere Art adverbialer Komponente annehmen, z. B. PERSON/SACHE sehen oder andere Verben der Wahrnehmung mit - aus dieser Perspektive analysiert - den grundlegenden Bedeutungen „(beginnen zu) sehen“ und „sehen“ selbst. Siehe:

(24 a) PERSON/SACHE sehen

(24 b) beim Abbiegen sah ich ihn an der Ecke stehen

(24 c) auf dem Fest sah ich ihn in der Ecke stehen ${ }^{15}$

\footnotetext{
14 In diesem Zusammenhang ist es wichtig, die Relevanz der Anwendung bestimmter abtrennbarer Präverben (z. B. er-) zu beachten, wenn der Basis der Wert ZUSTWECHS oder ZUST zugeordnet wird. Siehe in diesem Zusammenhang Paare wie scheinen-erscheinen, wachen-erwachen, trinken-ertrinken, etc.

15 Dabei ist es wichtig die Varianten ZUST und ZUSTWECHS nicht mit den Werten INGRESS, PROZESS und EGRESS zu verwechseln, denn auch wenn sicherlich viele ingressive oder egressive Handlungen normalerweise einen Zustandswechsel mit einschließen
} 
(25 a) PERSON/SACHE hören

(25 b) mein Bruder hörte einen Knall

(25 c) mein Bruder hörte das Violinkonzert von Beethoven

Anhand dieser beider Beispielserien ist die Möglichkeit festzustellen, dass Verben wie sehen oder hören, je nach dem Wert der sie umgebenden möglichen Satzbestandteile adverbialer Natur (ZYBATOW, 1999: 68) oder der verbalen Handlung, mit welcher sie in Zusammenhang gesetzt werden könnten, mit jedweder der beiden festgelegten Kennzeichnungen verknüpft werden können. Zum Beispiel kann sehen in (24 b) der Wert ZUSTWECHS zugewiesen werden, bedingt durch die Punktualität, die die Existenz der adverbialen Komponente beim Abbiegen (in deren semantischer Mikrostruktur als [+ PUNKT] gekennzeichnet) dieser verleiht. Etwas ähnliches tritt in (25 b) auf, wo die Punktualität und dementsprechend die Zuweisung der Kennzeichnung ZUSTWECHS durch die Verwendung von einen Knall gegeben wird. Siehe dazu, beispielsweise mit Bezug auf (25):

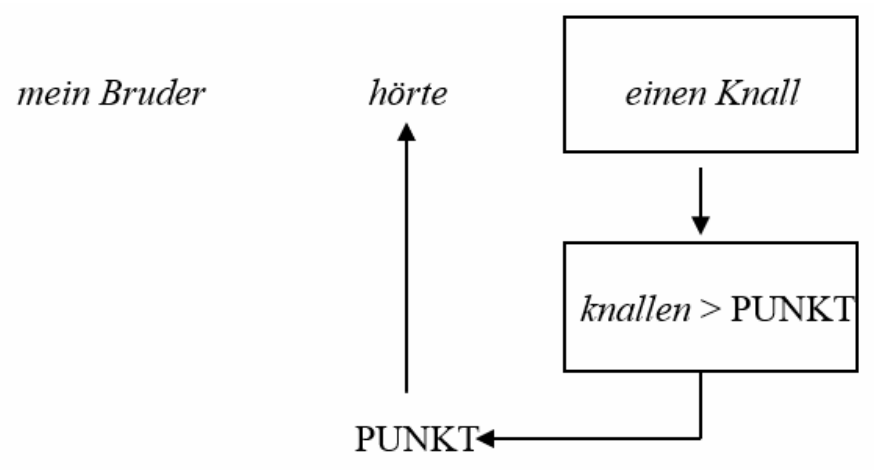

In $(24 \mathrm{c})$ und $(25 \mathrm{c})$ hingegen erweisen sich dieselben Verben als Träger des Wertes ZUST, im ersten Fall basierend auf dem von stehen dargestellten Bezug zu dem Inbegriff einer durativen Handlung und im zweiten Fall basierend auf der Einbeziehung des Objekts das Violinkonzert von Beethoven, das mit einer bestimmten Dauer, zumindest der des eigenen Werks des berühmten Komponisten, assoziiert wird.

und gleichermaßen viele durative Handlungen einen Zustand, gibt es - wie später dargelegt werden wird - viele, die diese Übereinstimmung nicht aufweisen. 
Die Zuordnung von (24 a) und (25 a) zu beiden Kennzeichnungen ergibt sich aus der Unmöglichkeit, im Rahmen der entsprechenden mit (b) gekennzeichneten Sätze und im Unterschied zu den mit (c) gekennzeichneten Sätzen den Kompatibilitätstest mit einer durativen Komponente durchzuführen, wie zum Beispiel mit der Adverbialen stundenlang. Siehe:

(26 a) beim Abbiegen sah ich ihn an der Ecke stehen

(26 b) * beim Abbiegen sah ich ihn an der Ecke stundenlang stehen

(27 a) auf dem Fest sah ich ihn an der Ecke stehen

(27 b) auf dem Fest sah ich ihn stundenlang an der Ecke stehen

(28 a) mein Bruder hörte einen Knall

(28 b) * mein Bruder hörte stundenlang einen Knall

(29 a) mein Bruder hörte das Violinkonzert von Beethoven

(29 b) mein Bruder hörte stundenlang das Violinkonzert von Beethoven

Diese mögliche doppelte Interpretation erlaubt jedwedem dieser Verben die Zuweisung der Werte ZUST oder ZUSTWECHS, die sich im besonderen Fall von sehen wie folgt darstellen würden:

SEHEN $=Z_{1}($ PERSON $/$ SACHE nicht sehen $)>Z_{2}($ PERSON $/$ SACHE sehen)

SEHEN $=Z_{1}($ PERSON $/$ SACHE sehen $)>Z_{2}($ PERSON $/$ SACHE sehen $)$

Neben dem bereits Ausgeführten und im Allgemeinen in Bezug auf die beiden behandelten signifikanten Achsen ist es wichtig, die semantische Autonomie dieser beiden hervorzuheben. Sie ist es, die es uns erlaubt, ihre jeweiligen Varianten unbegrenzt $\mathrm{zu}$ kombinieren und folglich vier verschiedene Modalitäten zu erhalten: „verursachte Zustandsänderung“ (KAUS ZUSTWECHS), „nicht verursachte Zustandsänderung“ (NICHT-KAUS ZUSTWECHS), „verursachter Zustand“ (KAUS ZUST) und „nicht verursachter Zustand“ (NICHT-KAUS ZUST). Als Beispiele für diese vier Varianten sind einige der produktivsten verbalen Komponenten bei der Bildung von Funktionsverbgefügen (FVG) in der deutschen Sprache erwähnenswert:

\begin{tabular}{|c|c|c|}
\hline & Zustandswechsel & Zustand \\
\hline Verursacht & bringen, führen, setzen, stellen, etc. & halten, behalten, etc. \\
\hline $\begin{array}{c}\text { Nicht } \\
\text { verursacht }\end{array}$ & gelangen, kommen, geraten, etc. & sein, bleiben, etc. \\
\hline
\end{tabular}


Die Auffassung der Gruppe bringen, führen, setzen, stellen, etc. oder der Gruppe halten, behalten, etc. als KAUS ergibt sich aus der Kausalität, die diese darstellen, gegenüber der NICHT-KAUS (gelangen, kommen, geraten, etc., oder sein, bleiben, etc.), wobei in diesem Sinne nicht wenige der Substantive als solche Nominalbestandteile des FVG - mit den vier betrachteten Varianten verwandt sind. So sehe man sich in diesem Zusammenhang zum Beispiel Ende (zu Ende bringen, zu Ende kommen, zu Ende halten, zu Ende sein) oder Gefahr (etwas in Bewegung setzen, in Bewegung kommen, etwas in Bewegung halten, in Bewegung sein) an:
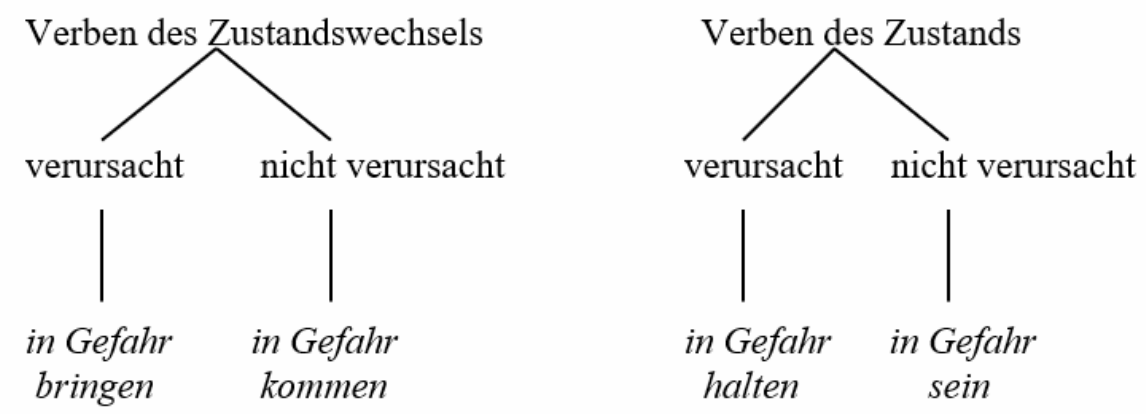

Im Hinblick auf die Bestimmung einiger der vier im Bereich der Kausalität festgelegten Varianten, ist gleichermaßen die weitreichende Produktivität der trennbaren Präverben als Elemente mit transformativer Fähigkeit in diesem Sinne zu erwähnen ${ }^{16}$. Erwähnenswert sind dabei die in der folgenden Tabelle dargestellten:

\begin{tabular}{|c|c|c|c|}
\hline $\begin{array}{c}\text { Kennzeichnung } \\
\text { KAUS }\end{array}$ & $\begin{array}{c}\text { Kennzeichnung } \\
\text { NICHT-KAUS }\end{array}$ & $\begin{array}{c}\text { Kennzeichnung } \\
\text { ZUSTWECHS }\end{array}$ & $\begin{array}{c}\text { Kennzeichnung } \\
\text { ZUST } \\
\end{array}$ \\
\hline $\begin{array}{c}\text { an- } \\
\text { lügen (JD) > } \\
\text { anlügen (JD, JDN) }\end{array}$ & $\begin{array}{c}\text {-[an-] } \\
\text { anlügen (JD, JDN) } \\
>\text { lügen }(\mathrm{JD})\end{array}$ & $\begin{array}{c}\text { er- } \\
\text { blühen (JD, DAUER) > } \\
\text { erblühen (JD, PUNKT) }\end{array}$ & $\begin{array}{c}-[\mathrm{er}-] \\
\text { erblühen (JD, PUNKT) } \\
>\text { blühen (JD, DAUER) }\end{array}$ \\
\hline $\begin{array}{c}\text { be- } \\
\text { wohnen (DD, EST) > } \\
\text { bewohnen (JD, ETW) }\end{array}$ & $\begin{array}{c}-[\mathrm{be}-] \\
\text { bewohnen (DD, ETW) } \\
>\text { wohnen (DD, EST) }\end{array}$ & & \\
\hline
\end{tabular}

16 In diesem Sinne werden die Präverben nicht nach der Art ihres jeweiligen Prozesses der Wortzusammensetzung (Ableitung oder Zusammensetzung), nach der lexikalischen Kategorie, mit der sie identifiziert werden (Präverben auf der Basis von Adjektiven, Adverbien, Präpositionspräverben usw.), oder, im Bereich der Präverben, die präpositional-adverbialen Charakter aufweisen, nach der Funktion, die ihnen zugeordnet werden kann, unterschieden. 
Aus dieser Tabelle entnehmen wir die Entkoppelung der beiden innerhalb der kausalen Dimension behandelten Unterachsen KAUS / NICHT-KAUS und ZUSTWECHS / ZUST, insofern als dass die Berücksichtigung einer von ihnen in der ersten, diese nicht mit der zweiten verknüpft oder umgekehrt. In diesem Zusammenhang ist die Relevanz des Vorhandenseins oder Nichtvorhandenseins von an bei der Definition der Kennzeichen KAUS / NICHT-KAUS zu beachten, da dies in Bezug auf die Dimension ZUSTWECHS / ZUST völlig irrelevant ist. Gleiches gilt für er beim Bestimmen des Kennzeichens ZUSTWECHS, was in Bezug auf KAUS / NICHT-KAUS nicht von Relevanz ist.

Um eine richtige Behandlung der beiden innerhalb der kausalen Achse erörterten kognitiven Unterdimensionen ausführen zu können, muss das Verb, welches Gegenstand der Analyse ist, im sogenannten „kausalen Wirkungsbereich " 17 des besagten Verbs ${ }^{18}$ verortet werden. Dieser wird durch den vollständigen lexikalischen Korpus einbezogen, der sich aus der Kombination der verschiedenen festgelegten Varianten ergibt ${ }^{19}$. Beispielsweise kann jedem als NICHT-KAUS markierten Verb die Kennzeichnung KAUS zugewiesen werden, indem grammatikalische Komponenten unterschiedlichster Art verwendet werden, wie etwa das AcI-Verb lassen (fahren > fahren lassen, schneiden > schneiden lassen), oder auch - wie in diesem Absatz bereits festgestellt - die vielen Präverben (lügen > anlügen, wohnen > bewohnen, etc. $)^{20}$. Genau die gleiche Relevanz wird der Auslassung

\footnotetext{
17 Diese „kausalen Wirkungsbereiche“ weisen zwei Unterschiede gegenüber den herkömmlichen „semantischen Wortfeldern“ auf. Ersterer bezieht sich auf die begrenzten Möglichkeiten der Strukturierung der „kausalen Wirkungsbereiche“, da diese in keinem Fall die für die Wortfelder typische Hierarchie in Makro-, Medio- und Mikrostruktur aufweisen. Letzterer ist die Möglichkeit, innerhalb der betreffenden „kausalen Wirkungsbereiche” nicht nur lexikalische Einheiten als solche, sondern auch jede andere Art von alternativen aspektischen Umschreibungen in Betracht zu ziehen.

18 Siehe dazu das Konzept der „Beherrschung“ (span.: „dominio“) von CUENCA / HILFERTY (1999: 97-124).

19 Im Zusammenhang mit diesem Beispiel ergibt sich der die „statische Positionierung“ betreffende kausale Wirkungsbereich aus den genannten Einheiten bringen / kommen / halten / sein.

${ }^{20}$ Diese Art der Wiederholung, die beim Erhalt kausativer Verben so häufig vorkommt, ist im umgekehrten Sinne nicht möglich, da jedes als KAUS markierte Verb kaum in NICHT-KAUS umwandelbar ist. Vgl. schweigen [NO CAUS] > schweigen lassen [CAUS] mit schlagen [CAUS] > -----). Der erste Fall, der mit NICHT-KAUS gekennzeichnet ist, lässt eine Erweiterung zu KAUS
} 
besagter Mechanismen bei der Kennzeichnung des Gegenteils, sprich NICHTKAUS-Handlungen (fahren lassen > fahren, anlügen > lügen, etc.) zuteil.

Ähnlich verhält es sich mit den Varianten ZUST / ZUSTWECHS, bei denen wir ebenfalls auf die (Nicht-)Anwendung von in dieser Hinsicht nützlichen grammatikalischen Mechanismen zurückgreifen - wieder einmal die Verwendung bestimmter Präverben (scheinen > erscheinen, etc.) - und auf das in jeder Sprache dafür zur Verfügung stehende Korpus - z. B. töten $>$ tot sein, usw. -, die alle in engem Zusammenhang mit den logisch-semantischen Möglichkeiten stehen, welche die Bedeutung des Verbs selbst bietet. Beispielhaft im Zusammenhang mit dem hier ausgeführten sind die Verben schlafen oder fallen, ihrerseits Prototypen der Aktionsarten ZUST und ZUSTWECHS. ${ }^{21}$

\section{KAUSALES THEMENFELD SCHLAFEN}

Variante KAUS / ZUSTWECHS

JDN einschlafen lassen ( $x$ verursacht $\left.[y \text { schläft ein }]_{\text {RESULTAT }}\right)$

JDN einschlafen lassen $\left[\mathrm{Z}_{1}\right.$ (y wacht) $>\mathrm{Z}_{2}$ (y schläft) $]$

Variante KAUS / ZUST

JDN schlafen lassen ( $x$ verursacht $\left.[y \text { schläft weiterhin }]_{\text {RESULTAT }}\right)$

JDN schlafen lassen $\left[Z_{1}\right.$ (y schläft) $>$ Z2 (y schläft)]

Variante NICHT-KAUS / ZUSTWECHS

einschlafen ([x schläft von selbst ein $]$ )

einschlafen $\left[Z_{1}(x\right.$ ist wach $)>Z_{2}(x$ schläft $\left.)\right]$

zu (schweigen > schweigen lassen). Der zweite Fall lässt im Gegenteil keine Modifikation in umgekehrter Richtung zu.

${ }_{21}$ Wie bereits im vorigen Abschnitt im Hinblick auf die Möglichkeit, den Ingress einer ingressiven Handlung, den Egress einer egressiven Handlung oder sogar die Iteration einer bereits iterativen Handlung festzustellen, bestimmt wurde, ist die Möglichkeit hervorzuheben, Änderungen an bereits mit diesem Merkmal gekennzeichneten Basen vorzunehmen, um auch in diesem Fall Varianten vom Typ KAUS zu erhalten. Dies kann nur selten durch die Anwendung einer lexikalischen Einheit mit einer solchen Funktion erreicht werden, sodass in fast allen Fällen auf einen der bereits etablierten Mechanismen oder eine Kombination davon zurückgegriffen werden muss. Siehe dazu Frank stirbt > Peter tötet Frank > Karl lässt Peter Frank töten oder auch Frank geht einkaufen > Peter lässt Frank einkaufen gehen > Karl lässt Peter Frank einkaufen gehen lassen. 
Variante NICHT-KAUS / ZUST

schlafen ([x schläft $]$ )

schlafen $\left[Z_{1}\right.$ (x schläft $)>Z_{2}$ (x schläft $\left.)\right]$

\section{KAUSALES THEMENFELD FALLEN}

Variante KAUS / ZUSTWECHS

ETWAS fallen lassen ( $x$ verursacht $[y \text { fällt }]_{\text {RESULTAT }}$ )

ETWAS fallen lassen $\left[\mathrm{Z}_{1}\right.$ (y steht $)>\mathrm{Z}_{2}$ (y fällt $\left.)\right]$

Variante KAUS / ZUST

ETWAS DAUERHAFT fallen lassen ( $x$ verursacht [ $y$ fällt $]_{\text {RESULTAT) }}$

ETWAS DAUERHAFT fallen lassen $\left[\mathrm{Z}_{1}\right.$ (y fällt $)>\mathrm{Z}_{2}$ (y fällt $\left.)\right]$

Variante NICHT-KAUS / ZUSTWECHS

fallen ( $x$ fällt von selber)

fallen $\left[\mathrm{Z}_{1}(\mathrm{x}\right.$ steht $)>\mathrm{Z}_{2}$ ( $\mathrm{x}$ fällt $\left.)\right]$

Variante NICHT-KAUS / ZUST

DAUERHAFT fallen ( $\mathrm{x}$ fällt beständig von selber)

DAUERHAFT fallen $\left[Z_{1}(x\right.$ fällt $)>Z_{2}$ ( $x$ fällt $\left.)\right]$

Ausgehend von diesen beiden NICHT-KAUS-Verben ist festzustellen, wie einfach es ist, die jeweilige KAUS-Variante zu erhalten, sei es durch Verwendung eines anderen alternativen Verbs, wie es im Fall von schlafen zur Verfügung stünde, oder - in letzter Instanz - durch Verwendung des AcIVerbs lassen. Anders ist der Ansatz, der die Varianten ZUST / ZUSTWECHS behandelt, da bei diesen in den speziellen Fällen durchaus lexikalische Einheiten in dieser Hinsicht bestehen (schlafen > einschlafen, etc.). Das „kausale Themenfeld“ dieser zwei Verben ergibt sich dementsprechend aus den folgenden Paradigmen:

\begin{tabular}{|c|c|c|}
\hline & ZUST & ZUSTWECHS \\
\hline KAUS & schlafen lassen & einschlafen lassen \\
\hline NICHT-KAUS & schlafen & einschlafen \\
\hline
\end{tabular}

\begin{tabular}{|c|c|c|}
\hline & ZUST & ZUSTWECHS \\
\hline KAUS & etwas fallen lassen & verursachen, dass etwas fällt \\
\hline NICHT-KAUS & fallen & von alleine ins Fallen geraten \\
\hline
\end{tabular}


Die deutlichsten Einschränkungen im Zusammenhang mit dem obigen Ansatz betreffen die Verben, die in Abschnitt 1.2. beim Erhalt der beiden mit ZUST bezeichneten Varianten als PUNKT gekennzeichnet wurden, da es sich diesmal um Verben handelt, die Handlungen bezeichnen, die nicht zeitlich ausgedehnt werden können und daher prinzipiell mit dem ZUST-Wert unvereinbar sind. Eine gleichzeitige Einbeziehung beider Kennzeichnungen könnte nur im Sinne einer Herausarbeitung erfolgen, die nicht auf die Veränderung der Handlung selbst verweist - wie bei den Verben einschlafen oder auftauchen in (3) und (4) -, sondern auf den Zugang oder die Herangehensweise an eine solche Veränderung der Handlung oder auch auf die Einbeziehung irgendeiner Art von Iteration. Diesbezüglich eignet sich die Betrachtung eines Verbs mit diesen Eigenschaften, beispielsweise platzen:

\section{KAUSALES THEMENFELD PLATZEN}

Variante KAUS / ZUSTWECHS

ETWAS platzen lassen $\left(x\right.$ verursacht $[y \text { platzt }]_{\text {RESULTAT }}$

ETWAS platzen lassen $\left[\mathrm{Z}_{1}\right.$ (y ist nicht geplatzt) $>\mathrm{Z}_{2}$ (y ist geplatzt) $]$

Variante KAUS / ZUST

JDN DAUERHAFT platzen $\left(x\right.$ verursacht $[y \text { platzt DAUERHAFT }]_{\text {RESULTAT }}$

JDN DAUERHAFT platzen [Z1 (y platzt) $>$ Z2 (y platzt)]

Variante NICHT-KAUS / ZUSTWECHS

platzen (x platzt von selber)

platzen $\left[Z_{1}(x\right.$ ist nicht geplatzt $)>Z_{2}(x$ ist geplatzt $\left.)\right]$

Variante NICHT-KAUS / ZUST

DAUERHAFT platzen (x platzt DAUERHAFT von selber)

DAUERHAFT platzen $\left[\mathrm{Z}_{1}(\mathrm{x}\right.$ platzt $)>\mathrm{Z}_{2}(\mathrm{x}$ platzt $\left.)\right]$

Im Hinblick auf ein punktuelles Verb wie platzen sei vor den enormen Schwierigkeiten gewarnt, die mit ZUST gekennzeichneten Varianten, d. h. die Kombination der Merkmale KAUS ZUST und NICHT-KAUS ZUST, $\mathrm{zu}$ erhalten. In diesen Fällen handelt es sich also, wie in (3) und (4) erneut festgestellt wird, weniger um einen ZUST-Wert als vielmehr um einen möglicherweise verlängerten Zugang zum Zustandswechsel als solchem oder, falls notwendig, um eine Iteration, wie in (30) bzw. (31): 
(30) du machst so einen Lärm, dass meine Ohren platzen

(31) die ganze Nacht ließen die Kinder Ballons auf der Party platzen

Bei (30) sei bedacht, dass sich die andauernde Bedeutung des Verbs nicht aus der Handlung platzen selbst ergibt, bei der es sich um eine PUNKT-Handlung handelt, sondern vielmehr aus dem Prozess, der tatsächlich in zeitlicher Hinsicht ausdehnbar ist. Die andauernde Bedeutung in (31) ist im Gegensatz dazu ausschließlich mit der mittels der adverbialen Komponente die ganze Nacht implizit geäußerten Iteration verknüpft.

\section{Kategoriale Kollisionen}

Die Berücksichtigung der drei betrachteten Koordinaten - temporal, kausal und aspektuell - kommt zu einer Anordnung eines breiten terminologischen Spektrums, das traditionell mit der sogenannten „Aktionsart“ verbunden ist. Es handelt sich um drei Bereiche mit - von wenigen Ausnahmen abgesehen, wie bereits im Zusammenhang mit den Merkmalen von ZUSTWECHS und DURAT erläutert wurde - vollständiger funktioneller und semantischer Autonomie, obwohl in dieser Hinsicht besonders die Unterschiede zwischen einigen der festgestellten Werte beachtet werden müssen, da es in diesen Fällen eine Tendenz zu einer nicht ganz gerechtfertigten Art und Weise der Verbindung gibt.

Die Werte PUNKT und ZUSTWECHS. Wie bereits herausgearbeitet, gilt für die Gesamtheit der PUNKT-Verben notwendigerweise auch die Kennzeichnung ZUSTWECHS - sofern die Kürze, mit der die verbale Handlung stattfindet (man erinnere sich an Verben des Typs platzen, finden usw.), eine unmittelbare Kulmination der beschriebenen Handlung bewirkt -, obwohl dabei betont sei, dass dieser Zusammenhang in entgegengesetzter Richtung nicht besteht und es eine große Anzahl von ZUSTWECHS-Verben gibt, die z. B. als DURAT klassifiziert werden können. Als Beispiel dient irgendeines der obigen Verben, die sich auf den Bereich der Positionierung beziehen (setzen, legen, stellen, etc.) oder eines derjenigen, die mit den räumlichen Unteraktionen öffnen/schließen in Zusammenhang stehen. All diese drücken eine Zustandsänderung aus, können dabei aber immer noch die Variable DURAT aufzeigen. Dabei ist für einige der ersten Gruppe, z. B. legen, auf die Analyse $\mathrm{Z}_{1}$ [nicht liegen (stehen, etc.)] $>\mathrm{Z}_{2}$ (liegen) zurückzugreifen, oder in Bezug auf einige der zweiten, z. B. öffnen, auf die Analyse $Z_{1}$ (zu sein) > $Z_{2}$ (auf sein). Obwohl es sich bei ihnen also um 
in ihrer Ausführung kurze Aktionen handelt, können sie manchmal eine gewisse Dauer aufweisen, wie aus Kontextualisierungen wie die zwei Tonnen schwere Tür ist sehr schwer zu öffnen etc. ersichtlich ist.

Die Werte DURAT PROZESS und DURAT INGRESS / DURAT EGRESS. Diese Kollisionen treten um Zusammenhang mit den so genannten Strukturen „zweipoliger Gegensätze“ auf, die in der deutschen Sprache besonders häufig in Form der Verbpaare aufmachen / zumachen, anmachen / ausmachen, etc. auftreten. Auf der Kausalachse handelt es sich um sechs Fälle von ZUSTWECHS-Handlungen, während sie auf der Temporalachse nicht notwendigerweise als DURAT-INGRESS oder DURAT-EGRESS eingeordnet werden würden, sondern viel eher als DURAT-PROZESS. Dies ist auf den Bezug zurückzuführen, den die Bedeutung eines jeden der genannten Verben mit der vollständigen Handlung herstellt. Es wurde diesbezüglich bereits der PROZESS-Wert festgelegt, der in nicht wenigen Fällen den als ZUSTWECHS gekennzeichneten Verben zugeordnet werden kann. Unabhängig davon erweist es sich entsprechend den Nummern (32) und (33) als notwendig, die Handlungen neu $\mathrm{zu}$ formulieren, um ihnen die Kennzeichnung DURAT INGRESS oder DURAT EGRESS zuweisen zu können:22

(32) wir haben angefangen, die Tür aufzumachen

(33) wir haben damit aufgehört, die Tür aufzumachen

Die Werte IMPERF und DURAT / PERF und INGRESS, PERF und EGRESS. Die Variablen PUNKT / DURAT bedingen nicht das Auftreten des einen oder anderen Aspekts, da es sich im diesem letzten Fall um eine grammatikalische Kategorie an sich handelt. Es sind die Verwendungen zu beachten, welche die beiden innerhalb der zwei, in der Zeitachse festgelegten Variablen zeigen:
der Staatschef ist gestorben
(PUNKT - PERF)
gerade als der Staatschef starb, ...
(PUNKT - IMPERF)
mein Vater ist nach Madrid gefahren
(DURAT - PERF)
gerade als mein Vater nach Madrid fuhr, ...
(DURAT - IMPERF)

${ }^{22}$ Gleichermaßen im Bezug auf die Präverben ein - aus (eingießen - ausgießen, einladen -
ausladen, etc.), auf- ab (aufbauen - abbauen, aufsteigen - absteigen, etc.), etc. zu sehen. 
Wenn dem verbalen Aspekt ein der zeitlichen Dimension fremder kognitiver Bereich zugewiesen wird, ergeben sich die Werte PUNKT und IMPERF nicht als miteinander unvereinbar, da die Verwendung des zweiten nur die bestehende Relativität zwischen einer Handlung A und einer anderen B zeigt, um die temporale Gleichzeitigkeit beider festzustellen.

\section{Schlussfolgerungen}

Gemäß dem in dieser Arbeit dargelegten, sind die folgenden grundlegenden Schlussfolgerungen hervorzuheben:

1. Angesichts der großen Menge der über die Möglichkeiten, die AA der (deutschen) Verben a priori festzulegen formulierten Meinungen, und vor allem wegen des Mangels an stabilen Kriterien, halten wir eine Vereinheitlichung der bestehenden Nomenklatur sowie eine Ordnung der Grundwerte nach einigen zuvor festgelegten semantischen Kriterien für notwendig. Zu diesem Zweck treten wir für die Existenz zweier Arten unabhängiger Deskriptoren ein, die sich auf die kausale und zeitliche Dimension jedweder Handlung beziehen, die Gegenstand der Analyse sein könnte.

2. Die Analyse der AA im Rahmen der zeitlichen Dimension umfasst die zwei grundlegende Varianten PUNKT / DURAT, innerhalb derer eine Unterscheidung der Unterarten INGRESS, PROZESS und EGRESS möglich ist. Innerhalb beider Achsen können diese drei Varianten sich zusätzlich als ITERAT / NICHT-ITERAT darstellen, weswegen in diesem Sinne zwischen zwölf Varianten unterschieden wird: iterative ingressive punktuelle Verben, nicht-iterative ingressive punktuelle Verben, iterative prozessuale punktuelle Verben, nicht-iterative prozessuale punktuelle Verben, iterative egressive punktuelle Verben, nicht-iterative egressive punktuelle Verben, iterative ingressive durative Verben, nicht-iterative ingressive durative Verben, iterative prozessuale durative Verben, nicht-iterative prozessuale durative Verben, iterative egressive durative Verben und nicht-iterative egressive durative Verben.

3. All diese Verben unterwerfen sich einer Analyse kausaler Art, entsprechend der Kennzeichen ZUST / ZUSTWECHS sowie KAUS / NICHT-KAUS, wodurch in diesem Sinne vier verschiedene Kombinationen 
erhalten werden: Verben des verursachten Zustandswechsels (bringen, setzen, stellen, etc.), Verben des nicht verursachten Zustandswechsels (kommen, gehen, gelangen, geraten, etc.), Verben des verursachten Zustands (halten, beibehalten, etc.) und Verben des nicht verursachten Zustands (sein, bleiben, sich befinden, etc.). Jede dieser Kombinationen ist nicht nur auf die Verbalbase als solche anwendbar, sondern auch auf die Anordnung der FVG. In diesem Kontext ist beispielsweise der Zusammenhang von etwas in Bewegung setzen, in Bewegung kommen, etwas in Bewegung halten, in Bewegung sein, etc. zu sehen.

4. Die kausalen und temporalen Koordinaten können im Gegensatz zum Aspekt, der in jedem Fall eine Anwendung seiner beiden Varianten (perfekt / imperfekt) zulässt, da er selbst eine verbale grammatikalische Kategorie ist, a priori abgegrenzt werden.

\section{BIBLIOGRAFIE}

Andersson, S. G. (1972). Aktionalität im Deutschen. Band 1. Uppsala.

Ballweg, J. (1977). Semantische Grundlagen einer Theorie der deutschen kausativen Verben. Tübingen.

Balzer, B. (1999). Gramática funcional del alemán, Madrid, Ediciones de la Torre.

Bierwisch, M. (1989). Event Nominalisation: Proposals and Problems. In: Motsch, W. (Hrg.) Wortstruktur und Satzstruktur. Linguistische Studien, Reihe A. Berlin: Akademie der Wissenschaften, S. 1-73.

Binnik, R. I. (1991). Time and the Verb (A Guide to Tense and Aspect). Oxford University Press.

Cohen, D. (1989). El aspecto verba. Madrid: Visor.

Coseriu, E. (1986). Principios de semántica estructural. Madrid: Gredos.

Cuenca, M. J. \& Hilferty, J. (1999). Introducción a la lingüística cognitiva. Barcelona: Ariel.

Davidson, D. (1990). Handlung und Ereignisse. Frankfurt am Main: Suhrkamp.

Egg, M. (1994). Aktionsart und Kompositionalität. Berlin: Akademie Verlag.

Fabricius-Hansen, C. (1975). Transformative, intransformative und kursive Verben. Tübingen: Niemeyer.

Fefilov, A. I. (1981). Die Rolle der Aktionalen Kohärenz bei der textfragmentarischen Wiedergabe der Fortbewegungsarten, DaF 18, S. 14-151.

Flämig, W. (1965). Zur Funktion des Verbs. III. Aktionsart und Aktionalität, DaF 2, S. 4-12.

Gierden, C. (2000). El subjuntivo alemán. Teoría y práctica para hispanohablantes. Universität Valladolid.

Gross, H. (1974). Der Ausdruck des Verbalaspekts in der deutschen Gegenwartssprache. Hamburger phonetische Beiträge. Hamburg: Helmut Buske.

Kiefer, F. (1992). Aspect and conceptual structure: The progressive and the perfective in Hungarian, In: Zimmermann, I.-Strigin, A. (eds): Fügungspotenzen, Studia Grammatica 34. Berlin: Akademie Verlag, S. 89-110. 
Kleiber, G. (1995). La semántica de los prototipos. Categoría y sentido léxico. Madrid: Visor.

Krifka, M. (1989). Nominalreferenz und Zeitkonstitution: Zur Semantik von Massentermen, Pluraltermen und Aspektklassen. München: Wilhelm Fink.

López-Campos Bodineau, R. (1998). Los preverbios separables en lengua alemana. Sevilla.

Lyons, J. (1977). Semántica. Barcelona: Teide

Renicke, H. (1950). Theorie der Aspekte und Aktionsarten, Beiträge zur Geschichte der deutschen Sprache und Literatur 72, S.150-193.

Russinova, M. V. (1976). Die Präposition an als Komponente beim Ausdruck der Aktionsarten des Verbs, DaF 13, S. 119-121.

Steinitz, R. (1981). Der Status der Kategorie "Aktionsart“ in der Grammatik (oder: Gibt es Aktionsarten im Deutschen?). Berlin: Akademie der Wissenschaften.

Stiebels, B. (1994). Lexikalische Argumente und Adjunkte. Zum semantischen Beitrag von verbalen Präfixen und Partikeln, Dissertation, Heinrich-Heine-Universität Düsseldorf.

Storsch, G. (1978). Semantische Untersuchungen zu den inchoativen Verben im Deutschen. Braunschweig.

Trost, K. (1977). Verbalaspekt und Satzaspekt. Thesen zum Aspektcharakter von Aktiv und Passiv im Russischen unter Berücksichtigung des Deutschen, Sprachwissenschaft 2, S. 1-26.

Vater, H. (1994). Einführung in die Zeit-Linguistik. Köln: Gabel

Zybatow, T. (1999). Temporale Eigenschaften der Verben und das Perfekt. Leipzig. 